\title{
Flexible Platinum Temperature Sensor Embedded in Polyimide Films for Curved Surface Temperature Monitoring Applications: Skin Temperature of Human Body
}

\author{
Joon-Shik Park, ${ }^{*}$ Dong-Su Lee, ${ }^{1}$ Hyun Woo Nho, \\ Dong-Sun Kim, Tae-Ho Hwang, and Nak-Kyu Lee ${ }^{2}$ \\ Korea Electronics Technology Institute, Seongnam, Kyunggi, 13509, Republic of Korea \\ ${ }^{1}$ GMEK Inc., Anyang, Kyunggi, 431-767, Republic of Korea \\ ${ }^{2}$ Korea Institute of Industrial Technology, Ansan, Kyunggi, 15588, Republic of Korea \\ (Received February 17, 2017; accepted April 28, 2017)
}

Keywords: flexibility, Pt, thin film, polyimide, curved surface, skin temperature, sensor

In this paper, we describe the design, fabrication, and characterization of a flexible platinum temperature sensor embedded in polyimide (PI) films for temperature monitoring applications on curved surfaces, such as the skin of a human body, and various tubes and pipes. In order to optimize and enhance the properties of the flexible temperature sensors, we investigated the effects of the designed thickness $(0.5,1.0$, and $1.5 \mu \mathrm{m})$ and length $(10,50,100$, and $150 \mathrm{~mm})$ of Pt thin-film electrodes embedded in PI films on the temperature coefficient of resistance (TCR) properties and sensitivities. The average TCRs for flexible temperature sensors with different lengths but identical thicknesses of Pt thin-film electrodes were $0.0027,0.0029$, and $0.0030{ }^{\circ} \mathrm{C}^{-1}$ for designed thicknesses of $0.5,1.0$, and $1.5 \mu \mathrm{m}$ of Pt thin-film electrodes, respectively. The TCRs slightly increased when the thickness of the Pt electrodes increased. Meanwhile, thinner or longer Pt electrodes led to higher sensitivity for the temperature sensors. The highest sensitivity of a fabricated flexible temperature sensor was $15.59 \Omega \Omega^{\circ} \mathrm{C}^{-1}$ in the device with a length of $150 \mathrm{~mm}$ and thickness of $0.5 \mu \mathrm{m}$. The bending effect on the change in the TCR of flexible temperature sensors, with radii of curvature of 5, 10, and $15 \mathrm{~mm}$, was small. In order to confirm the performance of the fabricated flexible temperature sensor, actual skin temperatures of the palm and back of the hand were measured.

\section{Introduction}

Temperature is one of the most important vital signals or physiological parameters of the human body. In cases of illness due to acute and chronic disease, body temperature is typically measured first. The body temperature of older and weaker patients especially should be monitored to avoid abrupt changes in health. Therefore, the continuous monitoring of body temperature using wearable flexible temperature sensors is important for keeping patients stable. Additionally, measuring the skin temperature is simpler and more practical for continuous and wearable monitoring than measuring the core temperature of the human body. In general, the skin temperatures, for example, from $28.9^{\circ} \mathrm{C}$ at the calf to $32.6{ }^{\circ} \mathrm{C}$ at the chest, ${ }^{(1)}$ are dependent upon

${ }^{*}$ Corresponding author: e-mail: jspark@keti.re.kr

http://dx.doi.org/10.18494/SAM.2017.1583

ISSN 0914-4935 C MYU K.K. 
the position on the human body and are lower than the general core body temperature of $36.5^{\circ} \mathrm{C},{ }^{(1)}$ because of balance between heat production (core body) and heat loss (environmental condition). ${ }^{(2)}$

Recently, several research groups studied various types of flexible sensors for temperature sensing using Pt thin films ${ }^{(3-5)}$ and polymer films ${ }^{(6-9)}$ for wearable applications. There are both advantages and disadvantages in terms of the characteristics of temperature sensors using Pt thin films and polymer films. When using Pt thin films, the linearity and reproducibility are superior to those when using polymer films. However, the temperature coefficient of resistances (TCRs) of polymer films are much higher than those of Pt thin films even though polymer-filmbased temperature sensors show hysteresis behavior with increasing and decreasing temperature and relatively large deviations among polymer-based temperature sensors. Therefore, both Ptthin-film-based temperature sensors and polymer-film-based temperature sensors can be used in research to optimize and enhance the properties and create flexible and wearable applications. Additionally, temperature sensors using metal alloy ${ }^{(10,11)}$ or ceramic $^{(12-14)}$ were developed to obtain high TCR. However, those sensors were fabricated on rigid substrates.

In this study, we investigated the effects of the thickness and length of Pt thin films deposited on polyimide (PI) film with a thickness of $38 \mu \mathrm{m}$ on the TCR properties and sensitivities of flexible temperature sensors applied to skin temperature monitoring of the human body. From this study, we attempt to elucidate the optimal thickness and length of Pt-thin-film-based temperature sensors. Additionally, the Pt thin film was embedded in biocompatible PI films ${ }^{(15)}$ having a thickness of $6 \mu \mathrm{m}$ to avoid skin contact with Pt metal and the formation of biofilms from the human body on the Pt thin film.

\section{Experimental Procedure}

\subsection{Fabrication of flexible platinum temperature sensors embedded in PI films}

The fabrication process of the flexible temperature sensor is represented in Fig. 1. The device consisted of Pt thin film embedded in PI films for sensing the temperature on flexible PI. A glass wafer was used as a temporary carrier. The bottom PI film with a thickness of $38 \mu \mathrm{m}$ was laminated on the glass wafer. Pt thin films with thicknesses of $0.5,1$, and $1.5 \mu \mathrm{m}$ were deposited by a sputtering process and patterned on the PI film by the lift-off method, and 100-nm-thick Ti was used as an adhesion layer between Pt thin films and the PI film. Au pads were deposited on the Pt/Ti using a shadow mask. The top PI film with thickness of $6 \mu \mathrm{m}$ was spin-coated onto the wafer for the encapsulation of the Pt thin-film electrodes. Au pads were finally opened by reactive ion etching (RIE).

\subsection{Characterization of flexible platinum temperature sensors embedded in PI films}

Top images of the as-fabricated flexible temperature sensor were observed with an optical microscope and cross-sectional images were observed in a focused ion beam (FIB; FEI SCIOS) apparatus. In order to obtain TCR $(\alpha)$, the resistance $(R)$ of the Pt-thin-film-based flexible temperature sensor was measured in a silicone oil bath (RTE - 100LP, NESLAB) from 0 to 100 ${ }^{\circ} \mathrm{C}$ using a multimeter (HP 3458A, HP). A four-wire connection was used to eliminate the effect 


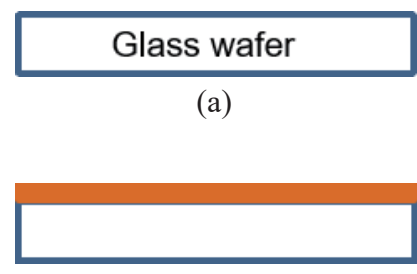

(b)

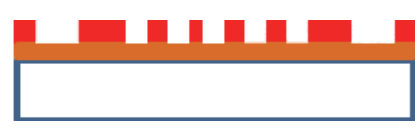

(c)

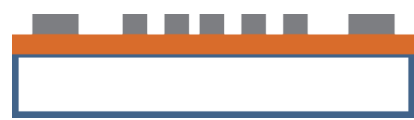

(d)

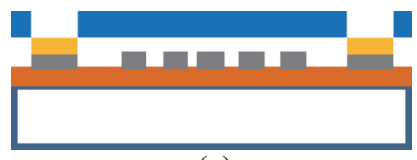

(e)

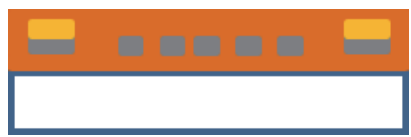

(f)

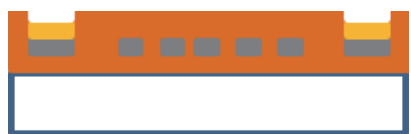

(g)

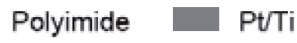

Photoresist Au

Shadow mask

Fig. 1. (Color online) Schematic illustration of fabrication process of flexible platinum temperature sensor embedded in PI film: (a) preparation of glass wafer as a temporary carrier, (b) lamination of PI $38 \mu \mathrm{m}$ thick on the glass wafer, (c) patterning of photoresist for deposited Pt/Ti thin film on PI, (d) deposition of Pt/Ti thin film by sputtering for temperature sensor, (e) deposition of Au pad using shadow mask, (f) spin coating of PI with thickness of $6 \mu \mathrm{m}$ for encapsulation, and (g) RIE process for Au pad opening.

of line resistance. TCR, indicating the percentage of change in the average resistance of the temperature sensor per ${ }^{\circ} \mathrm{C}$, is given by

$$
T C R=\Delta R /\left(R_{0} \cdot \Delta T\right)
$$

where $R_{0}$ is the resistance of the temperature sensor measured at an initial temperature of $0{ }^{\circ} \mathrm{C}$.

To clarify the bending effects of a flexible temperature sensor on resistance, we measured the resistance of the flexible temperature sensor on a hot plate (HP-20D, Daihan Scientific Co.) under three different bending conditions: to radii of curvature at 5,10 , and $15 \mathrm{~mm}$.

\section{Results and Discussion}

Photographs of the fabricated flexible temperature sensors are displayed in Fig. 2. We fabricated four types of devices with different lengths of serpentine Pt thin-film electrodes: $10 \mathrm{~mm}$ [(A-1) and (A-2)], $50 \mathrm{~mm}$ [(B-1) and (B-2)], $100 \mathrm{~mm}$ [(C-1) and (C-2)], and $150 \mathrm{~mm}$ [(D-1) and (D-2)]. On the front of the sensors [(A-1), (B-1), (C-1), and (D-1)], the exposed Au pads are visible as bright metallic colors. In the microscopic image (E) in Fig. 2, the width of the Pt thin-film electrode is confirmed as $15.1 \mu \mathrm{m}$, which is in agreement with the designed width of $15 \mu \mathrm{m}$.

Figure 3 shows that the actual thicknesses of fabricated flexible temperature sensors are different from designed values. Samples designed to have platinum electrodes $0.5,1.0$, and 1.5 $\mu \mathrm{m}$ thick and a Ti layer $100 \mathrm{~nm}$ thick had different actual thicknesses. The measured actual thicknesses of platinum electrodes are shown in Fig. 3(a): $347.42 \pm 20.61 \mathrm{~nm}$ and Ti layer thickness of $78.75 \pm 14.03 \mathrm{~nm}$, Fig. 3(b) $868.824 \pm 52.65 \mathrm{~nm}$ and Ti layer thickness of $103.97 \pm 18.29 \mathrm{~nm}$, and Fig. 3(c) $1270 \pm 69.42 \mathrm{~nm}$ and Ti layer thickness of $84.69 \pm 13.86 \mathrm{~nm}$. 


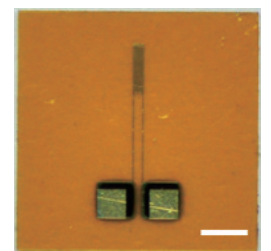

(A-1)

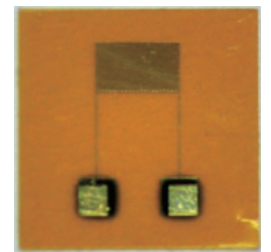

(B-1)

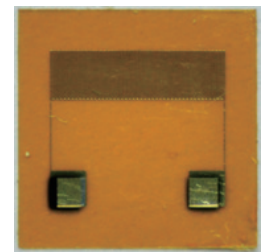

(C-1)
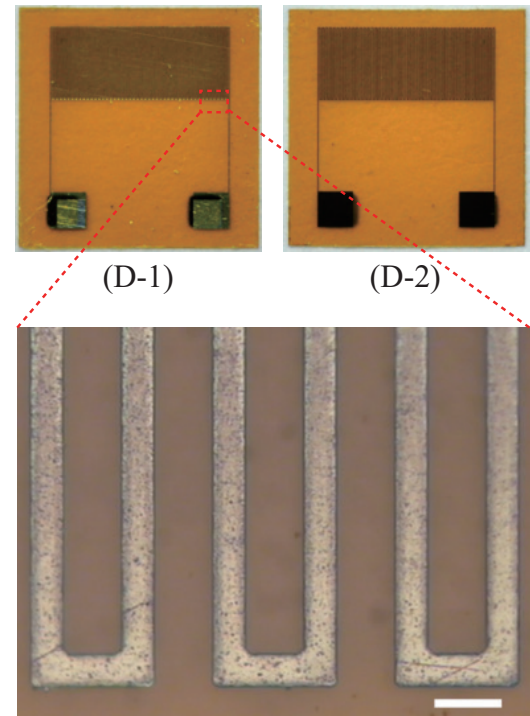

(E)

Fig. 2. (Color online) Photographs of front (A1-D-1) and back (A-2-D-2) of fabricated flexible temperature sensors with different lengths of Pt thinfilm electrodes. Lengths of Pt thin-film electrodes are (A-1) and (A-2) 10 (scale bar: $1 \mathrm{~mm}),(\mathrm{B}-1)$ and (B2) $50,(\mathrm{C}-1)$ and (C-2) 100, (D-1) and (D-2) $150 \mathrm{~mm}$ with width of $15 \mu \mathrm{m}$, and (E) optical microscopic image of the area marked in (D-1) (scale bar: $30 \mu \mathrm{m})$.

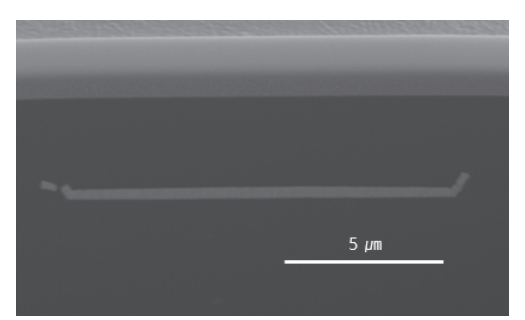

(a)

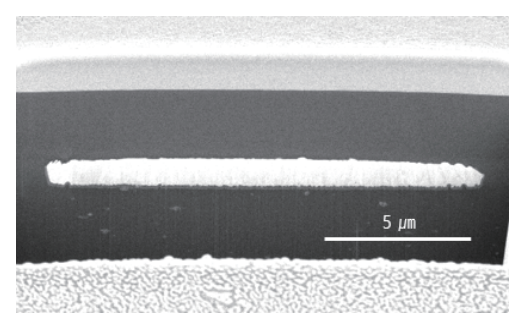

(b)

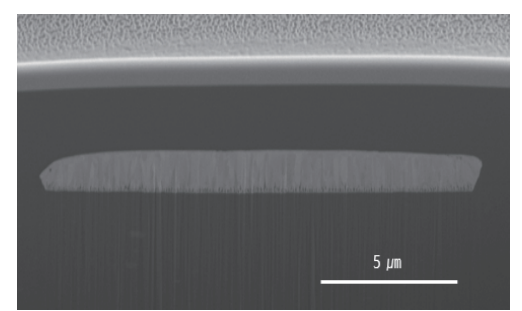

(c)
Fig. 3. Cross-sectional images of flexible temperature sensors designed with (a) 0.5-, (b) 1.0-, and (c) $1.5-\mu \mathrm{m}$-thick platinum electrodes with 100-nm-thick Ti layers (scale bar: $5 \mu \mathrm{m}$ ). 
The numerical values of resistance in Table 1 are plotted in Fig. 4. They show a resistance variation of flexible temperature sensors with various lengths and thicknesses of Pt thin-film electrodes. Experimental average resistances of devices with Pt thin-film electrodes having designed thicknesses of $0.5,1.0$, and $1.5 \mu \mathrm{m}$ are plotted with closed circles, open circles, and inverted triangles, respectively. Each value was line fitted for determining TCR, which is calculated from the slope and $y$-intercept. The TCR values calculated from data in Fig. 4 using Eq. (1) are listed in Table 2. From those results, we recognize that the effect of the thickness of Pt thin film on TCR is greater than that of length. When the thickness of Pt thin film was increased, there was a tendency for TCR to increase slightly. The average TCRs for flexible temperature sensors with different lengths but identical thickness of Pt thin-film electrodes were 0.0027, 0.0029 , and $0.0030{ }^{\circ} \mathrm{C}^{-1}$ for Pt thin-film electrodes with designed thicknesses of $0.5,1.0$, and 1.5 $\mu \mathrm{m}$, respectively. Even though these TCR values were slightly smaller than the value, 0.0039 ${ }^{\circ} \mathrm{C}^{-1}$, of bulk Pt, owing to the difference in the microstructures between thin-film Pt and bulk Pt, TCRs slightly increased with the thickness of Pt thin films from 0.5 to $1.5 \mu \mathrm{m}$. The sensitivity is a key parameter in addition to TCR for Pt-thin-film-based temperature sensors. The sensitivity is defined as the ratio of resistance change to temperature change, as shown by Eq. (2). The TCR is

Table 1

Measured resistances $(\Omega)$ at different temperatures from 0 to $100{ }^{\circ} \mathrm{C}$ in silicon oil. Standard deviations are denoted in parentheses. Types of flexible temperature sensors with different lengths of Pt thin-film electrodes: A $10 \mathrm{~mm}$ long, B $50 \mathrm{~mm}$ long, C $100 \mathrm{~mm}$ long, and D $150 \mathrm{~mm}$ long.

\begin{tabular}{lccccc}
\hline Device-designed thickness of & \multicolumn{5}{c}{ Temperature $\left({ }^{\circ} \mathrm{C}\right)$} \\
\cline { 2 - 6 } electrode $(\mu \mathrm{m})$ & 0 & 25 & 50 & 75 & 100 \\
\hline \multirow{2}{*}{ A-0.5 } & 390.85 & 416.86 & 444.78 & 471.02 & 497.65 \\
& $(10.09)$ & $(10.86)$ & $(12.39)$ & $(12.35)$ & $(14.55)$ \\
B-0.5 & 1929.35 & 2057.28 & 2192.17 & 2325.73 & 2456.91 \\
& $(13.46)$ & $(13.51)$ & $(14.27)$ & $(14.75)$ & $(16.82)$ \\
C-0.5 & 3862.46 & 4121.65 & 4387.93 & 4654.29 & 4916.07 \\
& $(28.61)$ & $(31.28)$ & $(31.27)$ & $(33.65)$ & $(39.49)$ \\
D-0.5 & 5672.91 & 6058.21 & 6451.44 & 6843.74 & 7228.33 \\
& $(14.25)$ & $(8.58)$ & $(6.42)$ & $(3.07)$ & $(1.59)$ \\
A-1.0 & 93.19 & 99.36 & 106.06 & 112.56 & 118.93 \\
& $(2.04)$ & $(1.87)$ & $(2.10)$ & $(2.15)$ & $(2.38)$ \\
B-1.0 & 455.80 & 488.16 & 522.22 & 555.56 & 587.90 \\
& $(8.87)$ & $(7.62)$ & $(7.81)$ & $(8.35)$ & $(11.25)$ \\
C-1.0 & 938.19 & 1002.18 & 1068.21 & 1136.53 & 1202.85 \\
& $(15.20)$ & $(15.23)$ & $(17.94)$ & $(18.96)$ & $(19.72)$ \\
D-1.0 & 1340.58 & 1437.20 & 1537.94 & 1638.07 & 1736.04 \\
& $(13.28)$ & $(12.27)$ & $(11.69)$ & $(12.31)$ & $(14.30)$ \\
A-1.5 & 89.34 & 95.86 & 102.64 & 108.96 & 116.09 \\
& $(1.67)$ & $(1.78)$ & $(1.87)$ & $(1.87)$ & $(2.31)$ \\
B-1.5 & 452.03 & 484.77 & 519.58 & 553.54 & 587.26 \\
C-1.5 & $(2.56)$ & $(2.83)$ & $(2.83)$ & $(3.11)$ & $(3.63)$ \\
& 916.38 & 981.86 & 1048.05 & 1115.53 & 1183.07 \\
D-1.5 & $(12.08)$ & $(9.94)$ & $(11.93)$ & $(10.57)$ & $(13.08)$ \\
& 1345.86 & 1444.67 & 1550.17 & 1650.96 & 1751.74 \\
& $(8.46)$ & $(7.01)$ & $(8.71)$ & $(7.34)$ & $(9.44)$ \\
\hline
\end{tabular}




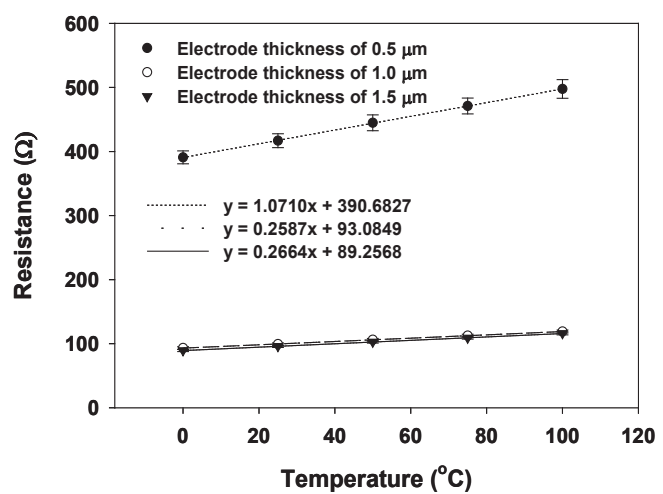

(a)

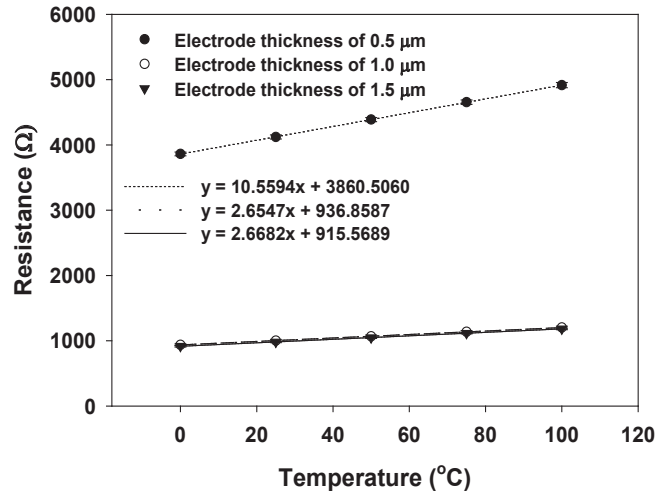

(c)

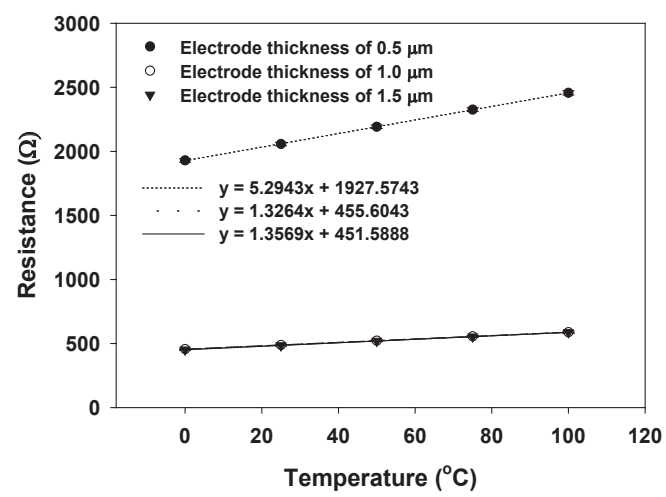

(b)

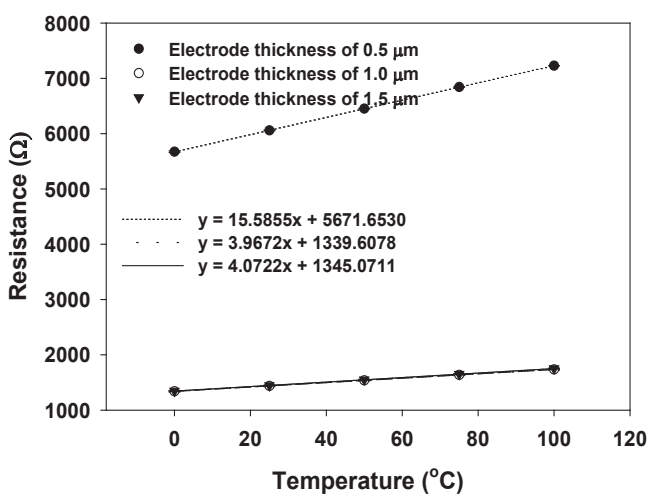

(d)

Fig. 4. Changes in resistance at different temperatures for flexible temperature sensors with different lengths of Pt thin-film electrodes: (a) 10, (b) 50, (c) 100, and (d) $150 \mathrm{~mm}$. Closed circles, open circles, and inverted triangles represent measured resistances of flexible temperature sensors with Pt thin-film electrodes having designed thicknesses of $0.5,1.0$, and $1.5 \mu \mathrm{m}$, respectively.

Table 2

Measured TCRs $\left({ }^{\circ} \mathrm{C}^{-1}\right)$ of various flexible temperature sensors with different lengths and thicknesses of Pt thinfilm electrodes. Each TCR is calculated from the slope and $y$-intercept in Fig. 4 using Eq. (1).

\begin{tabular}{lc}
\hline Device-designed thickness of electrode $(\mu \mathrm{m})$ & $\mathrm{TCR}\left({ }^{\circ} \mathrm{C}^{-1}\right)$ \\
\hline A-0.5 & 0.0027 \\
B-0.5 & 0.0027 \\
C-0.5 & 0.0027 \\
D-0.5 & 0.0027 \\
A-1.0 & 0.0028 \\
B-1.0 & 0.0029 \\
C-1.0 & 0.0028 \\
D-1.0 & 0.0030 \\
A-1.5 & 0.0030 \\
B-1.5 & 0.0030 \\
C-1.5 & 0.0029 \\
D-1.5 & 0.0030 \\
\hline
\end{tabular}


related to the sensitivity; however, it does not directly represent the amount of resistance change per ${ }^{\circ} \mathrm{C}$. Therefore, to prevent error, measuring the sensitivity is important.

$$
\text { Sensitivity }=\Delta R / \Delta T=T C R \cdot R_{0}
$$

As shown in Fig. 4, the sensitivity of the device (slope of graph) was affected by the thickness and length of $\mathrm{Pt}$ thin-film electrodes because sensitivity was affected by $R_{0}$. Therefore, the use of thinner or longer Pt thin-film electrodes to obtain higher $R_{0}$ led to a higher sensitivity of temperature sensors. These results concurred with the results obtained by Kim et al. ${ }^{(16)}$ The highest sensitivity of the fabricated flexible temperature sensors was $15.59 \Omega \Omega^{\circ} \mathrm{C}^{-1}$ in the device with a length of $150 \mathrm{~mm}$ and designed Pt thin-film electrode thickness of $0.5 \mu \mathrm{m}$.

Figure 5 shows the obtained electrical resistance ratio with increasing temperature from 0 to $100{ }^{\circ} \mathrm{C}$ in silicon oil. Using this result, the TCRs could be compared directly, because the slopes represent TCRs. Under these conditions, the TCRs were almost independent of the change in temperature, similar to the bulk-Pt-based resistance temperature detector. As shown in Fig. 1 and Table 1, the lengths of Pt thin-film electrodes were 10, 50, 100, and $150 \mathrm{~mm}$ in the cases of Figs. 5(a), 5(b), 5(c), and 5(d), respectively.

Figure 6 shows the effects of the thickness or lengths of Pt thin-film electrodes on $R_{0}$, which increased when the thickness of Pt thin-film electrode decreased or the length of Pt thin-film

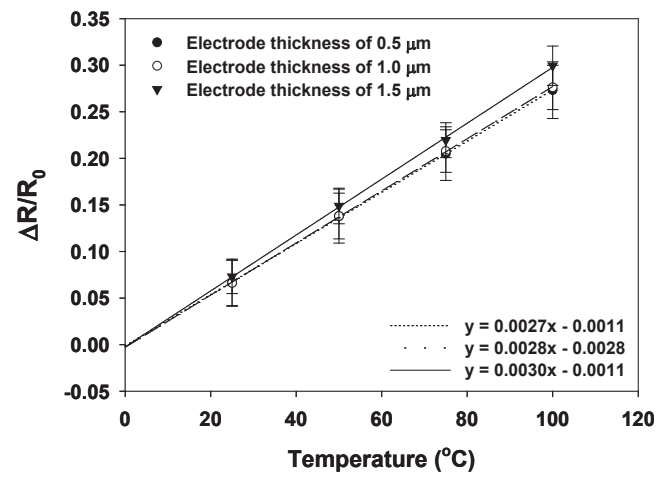

(a)

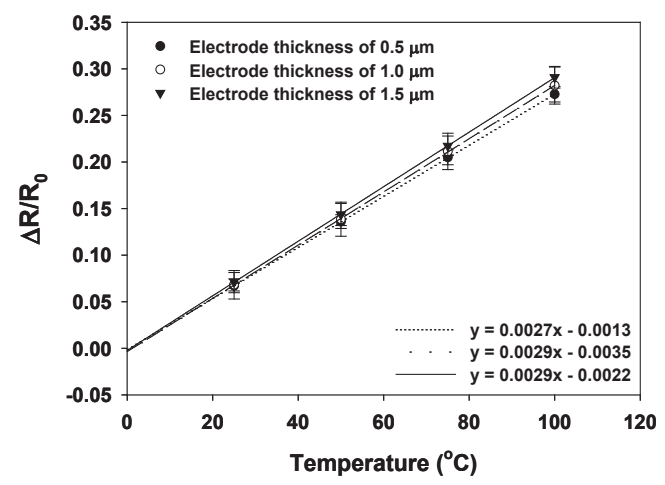

(c)

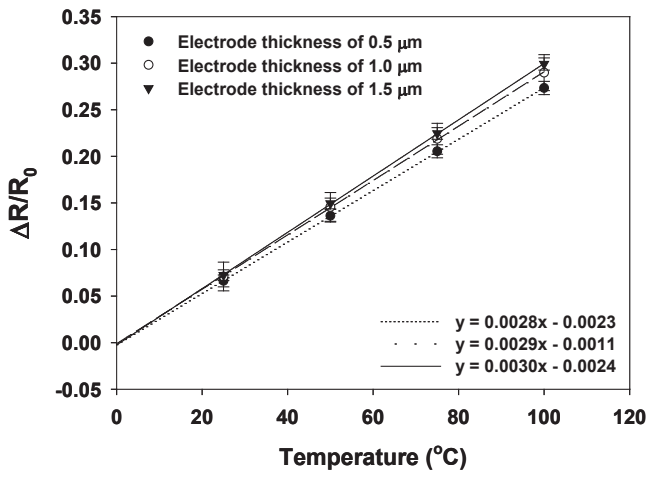

(b)

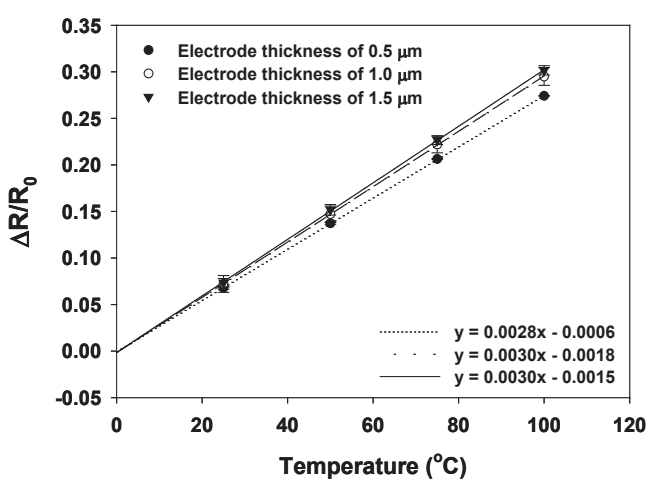

(d)

Fig. 5. Graphs of resistance ratios at different temperatures for flexible temperature sensors $150 \mathrm{~mm}$ thick with different lengths of Pt thin-film electrodes: (a) 10, (b) 50, (c) 100, and (d) $150 \mathrm{~mm}$. Closed circles, open circles, and inverted triangles represent measured resistances of flexible temperature sensors with Pt thin-film electrodes with designed thicknesses of $0.5,1.0$, and $1.5 \mu \mathrm{m}$, respectively. 


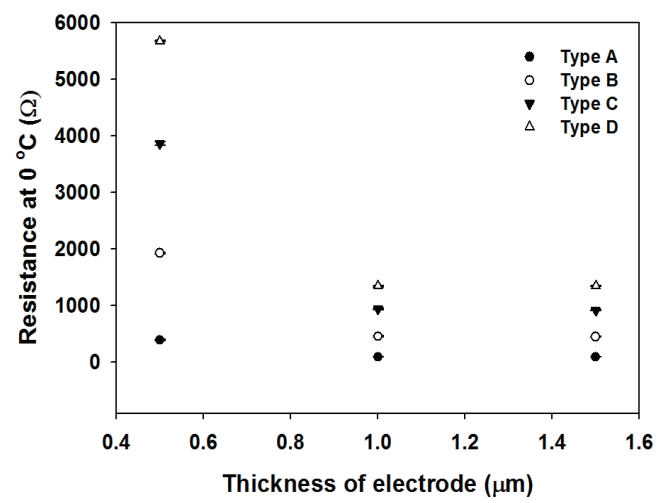

Fig. 6. Plot of resistances at $0{ }^{\circ} \mathrm{C}\left(R_{0}\right)$ versus thicknesses of $\mathrm{Pt}$ thin-film electrodes of flexible temperature sensors. 10-, 50-, 100-, and 150-mm-long Pt thin-film electrodes were denoted as Types A, B, C, and $\mathrm{D}$, respectively.

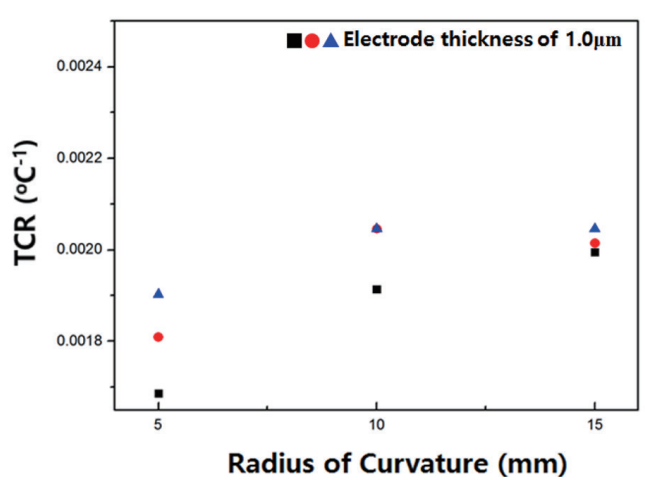

Fig. 7. (Color online) Change in TCR vs bending radius of curvature for flexible temperature sensors. The length and thickness of Pt thin-film electrode were $150 \mathrm{~mm}$ and $1.0 \mu \mathrm{m}$.

electrode increased. These results indicated significant differences in sensitivity between thin and thick electrodes, although the discrepancies among TCRs were not marked. Therefore, as represented by Eq. (2), the sensitivity was directly proportional to $R_{0}$. The percentage changes in resistance vs bending radius of curvature of 5,10 , and $15 \mathrm{~mm}$ of flexible temperature sensors were measured to confirm the possibility of application to slightly curved surfaces such as the palm and back of the hand or various tubes. In order to calculate TCR, temperatures were controlled on the hot plate.

As shown in Fig. 7, bending of three flexible temperature sensors with same dimensions had little effect on the variation in TCR. Therefore, we expect that fabricated flexible temperature sensors can be utilized for curved surfaces in the range of 5 to $15 \mathrm{~mm}$ radius of curvature.

In order to confirm the relevance of the fabricated flexible temperature sensor, actual skin temperatures of the palm and the back of the hand were measured and found to be 35.8 and 33.5 ${ }^{\circ} \mathrm{C}$, respectively.

\section{Conclusions}

We fabricated Pt-thin-film-based, mechanically flexible temperature sensors on PI film with a thickness of $38 \mu \mathrm{m}$. The flexible temperature sensors were designed and composed of serpentine Pt thin films with designed thicknesses of $0.5,1.0$, and $1.5 \mu \mathrm{m}$ and lengths of 10, 50, 100, and 150 $\mathrm{mm}$, respectively. The Pt thin films were encapsulated with 6 - $\mu \mathrm{m}$-thick PI to avoid direct skin contact with the Pt metal and the formation of biofilms on the Pt surface. The resistance of the fabricated Pt-based flexible temperature sensors exhibited good linear change with temperature variation. In addition, the TCR properties and sensitivities of the fabricated flexible temperature sensors were investigated to elucidate the effect of the thickness and length of the Pt thin films. The average TCRs for flexible temperature sensors with different lengths but identical thicknesses 
of Pt thin-film electrodes were $0.0027,0.0029$, and $0.0030{ }^{\circ} \mathrm{C}^{-1}$ for designed thickness of 0.5 , 1.0 , and $1.5 \mu \mathrm{m}$ of Pt thin-film electrodes, respectively. The highest sensitivity of the fabricated flexible temperature sensor was $15.59 \Omega \cdot{ }^{\circ} \mathrm{C}^{-1}$, which was obtained from the device with the thinnest $(0.5 \mu \mathrm{m}$ thick) and longest ( $150 \mathrm{~mm}$ length) $\mathrm{Pt}$ thin film. The bending effect on the change in the TCR of flexible temperature sensors with radii of curvature of 5, 10, and $15 \mathrm{~mm}$ was small. In order to confirm the performance of the fabricated flexible temperature sensor, actual skin temperatures of the palm and the back of the hand were measured.

\section{Acknowledgments}

We would like to acknowledge the financial support from the R\&D Convergence Program of the Ministry of Science, ICT and Future Planning and National Research Council of Science \& Technology of the Republic of Korea (Grant CAP-13-1-KITECH). Also, J.-S. Park thanks J. H. Bang for his helpful assistance in the measurement.

\section{References}

1 Korea Dictionary Research Publishing: Skin Temperature Test. The Great Encyclopedia of Nursing Science (1996).

2 Y. Houdas and E. F. J. Ring: Human Body Temperature: Its Measurement and Regulation (Springer, New York, 2013) p. 81.

3 S. Xiao, L. Che, X. Li, and Y. Wang: Microelectr. J. 38 (2007) 360.

4 A. Oprea, N. Bârsan, U. Weimar, J. Courbat, D. Briand, and N. De Rooij: Sensors IEEE Conf. (2007) p. 158.

5 D. H. Kim, S. Wang, H. Keum, R. Ghaffari, Y. S. Kim, H. Tao, B. Panilaitis, M. Li, Z. Kang, F. Omenetto, Y. Huang, and J. A. Rogers: Small 8 (2012) 3263.

6 T. Yokota, Y. Inoue, Y. Terakawa, J. Reeder, M. Kaltenbrunner, T. Ware, K. Yang, K. Mabuchi, T. Murakawa, M. Sekino, W. Voit, T. Sekitani, and T. Someya: Proc. Natl. Acad. Sci. 112 (2015) 14533.

7 J. Jeon, H. B. R. Lee, and Z. Bao: Adv. Mater. 25 (2013) 850.

8 W.-P. Shih, L.-C. Tsao, C.-W. Lee, M.-Y. Cheng, C. Chang, and Y.-J. Yang: Sensors 10 (2010) 3597.

9 J. S. Park, P. H. Kang, Y. C. Nho, and D. H. Suh: J. Appl. Polym. Sci. 89 (2003) 2316.

10 Y. Sato, T. Ishibashi, K. Obara, S. Ichida, Y. Morishita, and K. Sato: Jpn. J. Appl. Phys. 46 (2007) 297.

11 Y. V. Kudryavtsev and V. A. Oksenenko: J. Appl. Phys. 97 (2005) 113903.

12 L. N. Son, T. Tachiki, and T. Uchida: 37th Int. Conf. Infrared, Millimeter, and Terahertz Waves (2012).

13 Y.-H. Han, I.-H. Choi, H.-K. Kang, J.-Y. Park, K.-T. Kim, H.-J. Shin, and S. Moon: Thin Solid Films 425 (2003) 260.

14 Y. Lv, M. Hu, M. Wu, and Z. Liu: Surf. Coat. Technol. 201 (2007) 4969.

15 P. J. Rousche, D. S. Pellinen, D. P. Pivin Jr., J. C. Williams, R. J. Vetter, and D. R. Kipke: IEEE Trans. Biomed. Eng. 48 (2001) 361.

16 J. Kim, J. Kim, Y. Shin, and Y. Yoon: Korean J. Chem. Eng. 18 (2001) 61. 